\title{
Seroprevalence of IgG Anti-SARS-CoV-2 among Voluntary Blood Donors in Dubai: Demographic and Risk Factors
}

\author{
May Raouf Mariem Rabeh Suminder Kaur Ranjita Sharma \\ Nimmy Thottumkal Roukaya Mohammed \\ Dubai Blood Donation Center, Latifa Women and Children Hospital, Dubai Health Authority, \\ Dubai, United Arab Emirates
}

\section{Keywords}

SARS-CoV-2 antibody · Donor eligibility · Donor demography · Lifestyle

\begin{abstract}
Background and Objectives: During the pandemic of $\mathrm{CO}$ VID-19, additional blood donor eligibility criteria have been added to ensure safety of donors and donations. The aim of this study is to estimate the seroprevalence of SARS-CoV-2 antibody in eligible donors and to study the demography and lifestyle of asymptomatic cases. Materials and Methods: One thousand four hundred eighteen eligible donors were enrolled in this study in the Dubai Blood Donation Center from August to December 2020. Blood samples were tested for SARS-CoV-2 antibody. Donors' demographic characteristics and lifestyle were studied further. The $x^{2}$ test was used to analyze the data association. Results: $88.4 \%$ of the studied group were males, $85 \%$ were from age 17 to 45 years, and $53 \%$ were regular donors. The seroprevalence of SARSCoV-2 antibody was $13.5 \%$ and significantly related to gender, frequency of donation, collection site, type of donation, and more frequent when donors shared transport and ac-
\end{abstract}

karger@karger.com www.karger.com/dmj

Karger $\stackrel{\text { ' }}{5}$

GOPEN ACCESS
(C) 2021 The Author(s)

Published by S. Karger AG, Basel

This is an Open Access article licensed under the Creative Commons Attribution-NonCommercial-4.0 International License (CC BY-NC) (http://www.karger.com/Services/OpenAccessLicense), applicable to the online version of the article only. Usage and distribution for commercial purposes requires written permission. commodation. Blood group and age had no significant association. $5.5 \%$ were found to have cough/fever or a history of contact with positive COVID-19 patients. Conclusions: The seroprevalence of SARS-CoV-2 antibody was $13.5 \%$ among eligible blood donors, and hence the current eligibility criteria allow asymptomatic COVID-19 patients to donate blood. The seroprevalence was seen more in first time donors, suggesting that regular blood donors are safer. Additional risk factors related to lifestyle can be identified to ensure safety of transfusion during COVID-19 pandemic.

(c) 2021 The Author(s)

Published by S. Karger AG, Basel

\section{Introduction}

In late December 2019, China notified the WHO of a cluster of pneumonia cases with unknown etiology in the city of Wuhan [1]. By January 7, 2020, Chinese scientists had identified the pathogen as a novel coronavirus $[2,3]$. On January 30, the WHO Emergency Committee declared the COVID-19 outbreak a Public Health Emergency of International Concern [4] and on March 11, declared it a pandemic [5]. 
The infectious disease spread rapidly, reaching virtually every country in the world. As of December 2020, the cumulative numbers showed over 79 million reported cases and over 1.7 million deaths globally since the start of the pandemic [6].

SARS-CoV-2 is an enveloped virus in the genus Betacoronavirus, but it is significantly different from SARS$\mathrm{CoV}$ and MERS-CoV [7]. The infection often causes mild symptoms, including cough, muscle pain, and anosmia, and it can progress into high fever, pneumonia, respiratory distress [8], and, in some cases, death [9].

There is evidence that SARS-CoV-2 is transmissible by infected asymptomatic and presymptomatic individuals [10]. A number of transmission clusters with evidence of possible transmission from presymptomatic individuals in close contact have been reported [11]. In addition, SARS-CoV-2 RNA has been detected in respiratory swabs and feces from asymptomatic individuals [12].

Until now, there has been no solid evidence indicating that such respiratory viruses can be transmitted by blood transfusion $[13,14]$. However, considering the incubation period of SARS-CoV-2 infections (median, 5.2 days) and the fact that some can be asymptomatic, blood safety remains a general concern [15].

During the pandemic of COVID-19, the blood donation centers have adopted additional eligibility criteria to ensure safety of blood donors and patients receiving blood. The additional eligibility criteria have been continuously updated according to the information received from the WHO, Food and Drug Administration (FDA), and local health authorities in different countries.

The WHO has provided no recommendations about screening the donors for SARS-CoV-2 by RT-PCR or immunoassays; however, it recommends temporary deferral for 28 days if any symptoms (cough, fever, and flu) are present or if they are exposed to a confirmed COVID-19 patient or have traveled to an epidemic area. The WHO also recommends that the potential donors also have to inform the blood bank if they develop symptoms within 28 days of donation [14]. In March 2020, the American Association of Blood Banks (AABB) issued a toolkit [16] and further recommended on eligibility criteria and deferral guidelines [17].

Assessing the prevalence trends of viral infections among eligible blood donors is not only essential to estimate the effectiveness of blood safety strategies but also to improve the current strategies to increase transfusion safety, minimizing the potential risk of coronavirus by blood transfusion [15]. Studying the demography and lifestyles can help blood establishments to assess the cur- rent eligibility criteria to ensure the safety of blood especially during such a pandemic where lifestyle and personal behaviors have a major role in transmission of the infection.

Different countries have implemented different precautionary measures to avoid the transmission of coronavirus among their populations. In many countries, lockdown strategies and social distancing are mandatory to reduce person-to-person transmission [18]. Accordingly, the prevalence of asymptomatic cases varies in different countries, and this will be reflected in blood donor population.

In this study, we aimed to look at the seroprevalence of SARS-CoV-2 antibody in healthy voluntary and eligible blood donors and further study the demographic characteristics and lifestyle of the seropositive donors. This will help us to understand the effectiveness of our current eligibility criteria and identify additional risk factors which may support future decisions for safer blood transfusion.

\section{Materials and Methods}

A total of 1,418 eligible voluntary blood donors were enrolled in this study from random donors visiting the Dubai Blood Donation Center, between August and December 2020. The Dubai Blood Donation Center collects $50 \%$ of blood units in the United Arab Emirates. The center is accredited by the American Association of Blood Banks (AABB), and the donor eligibility criteria are frequently reviewed.

\section{Eligibility Criteria}

- When the COVID-19 pandemic started and after the WHO declaration of increasing number of COVID cases, we initiated revision of criteria for eligibility of donors at the end of January 2020. We started to defer for 28 days if any donor had a travel history outside the United Arab Emirates or a history of fever and acute symptoms of upper or lower respiratory illness and/ or had a close contact with a person who is under investigation for COVID-19. Those who had a positive PCR test were deferred for 3 months. In March 2020, as per the AABB toolkit [16], we revised and implemented updated deferral criteria. Then, in May 2020, as per the AABB toolkit, we again revised and implemented the following criteria related to COVID-19 [17].

- Cared for, lived with, or otherwise had close contact with individuals diagnosed with or suspected of having COVID-19 in the 28 days before donation.

- Been diagnosed with COVID-19 or who are suspected to have COVID-19 and who had symptomatic disease or had a positive diagnostic test (e.g., nasopharyngeal swab) for SARS-CoV-2 but never developed symptoms were deferred for 28 days after complete resolution of symptoms or the date of the positive diagnostic test, whichever period is longer. 
Table 1. Demographic characteristics and profile of 1,418 eligible donors

\begin{tabular}{llrr}
\hline Characteristic & Variables & \multicolumn{2}{l}{$\%$} \\
\hline Gender & Male & 1,262 & 88.38 \\
& Female & 156 & 10.92 \\
\hline Age, years & $17-35$ & 690 & 48.66 \\
& $36-45$ & 485 & 34.20 \\
& $46-65$ & 243 & 17.14 \\
\hline Nationality & Indians & 660 & 46.22 \\
& Emiratis & 211 & 14.78 \\
& Filipinos & 90 & 6.30 \\
& Pakistanis & 51 & 3.57 \\
& Others & 816 & 29.13 \\
\hline Site of donation & Donation center (fixed site) & 590 & 62.32 \\
& Campaigns/mobile bus & 661 & 37.68 \\
\hline Frequency of donation & First time donor & 757 & 53.29 \\
& Regular donor (>1 donation) & 1,211 & 85.40 \\
\hline Type of donation & Whole blood & 207 & 14.59 \\
\hline Blood group & Platelet apheresis & 592 & 41.75 \\
& O & 383 & 27.01 \\
& B & 357 & 25.18 \\
& A & 86 & 6.06 \\
\hline & AB & 100.00 \\
\hline
\end{tabular}

- Individuals who are tested and found positive for SARS-CoV-2 antibodies but who did not have prior diagnostic testing and never developed symptoms were allowed to donate without a waiting period and without performing a diagnostic test (e.g., nasopharyngeal swab).

The Dubai Blood Donation Center maintained safe environment to blood donors and employees: mandatory wearing of face masks, individual sanitization tunnels, social distancing, and a thermal camera for body temperature checking at the point of entrance (those with body temperature above $37.5^{\circ} \mathrm{C}$ were not allowed to enter the center).

In this study, the inclusion criteria for the participating donors were the fulfillment of donors' eligibility criteria [16, 17]. The prospective donor has to fill up the donor history questionnaire and undergo brief medical screening.

\section{Demographic, Lifestyle, and Risk Factors}

The demographic characteristics of the participating donors were derived from blood donors software - e.Delphyn. The following factors were considered: gender, age, nationality, site of donation (in the center or outdoor), frequency of donation (first time or regular donor [ $>1$ donation]), type of donor (whole blood or platelet apheresis), and blood group (ABO).

The participating donors who showed positive results for SARSCoV-2 IgG antibody were contacted by phone for further information concerning their lifestyle details and exposure risks for a period extending to 2 months prior to the current visit using a separate questionnaire which included the questions given in online suppl. material 1 (see www.karger.com/doi/10.1159/000517456 for all online suppl. material).

\section{Samples and Testing}

The plasma obtained from the donors' samples in EDTA tubes used for testing routine infectious disease markers was also used for testing SARS-CoV-2 antibody. Architect SARS-CoV-2 IgG chemiluminescent microparticle immunoassay (Abbott) was used for the qualitative detection of IgG antibodies to the nucleocapsid protein of SARS-CoV-2, in the donors' samples. Architect SARSCoV-2 IgG was granted FDA Emergency Use Authorization (PPV is $96.77 \%$ and NPV is $99.63 \%$ ).

The default result unit for the SARS-CoV-2 IgG assay is index $(\mathrm{S} / \mathrm{C})$, and the cutoff is 1.40 index $(\mathrm{S} / \mathrm{C})$. Sample index $<1.40$ is considered negative, and sample index $\geq 1.40$ is considered positive (manufacturer defined cutoff).

\section{Statistical Analysis}

The IgG antibodies to SARS-CoV-2 result of all units tested were tabulated in an Excel spreadsheet. For each unit, the demographic data were extracted from the e-Delphyn software, keeping the data anonymous. The tables and percentages were prepared using formulas within the Excel spreadsheet. Another Excel spreadsheet was prepared for listing the response of donors about their lifestyle, and then the data were analyzed and graphs were prepared.

The statistical association of the presence of IgG antibodies to SARS-CoV-2 to the demographic characteristics of the donors was checked using Epi Info ${ }^{\mathrm{TM}}, \mathrm{CDC}[19]$. The $\chi^{2}$ test at 5\% significance level was used to analyze the results. 
Table 2. Significance of IgG anti-SARS-CoV-2 test results

\begin{tabular}{|c|c|c|c|c|c|c|c|}
\hline Characteristics & Variables & Positive IgG & Negative IgG & Total tested & Positive, $\%$ & $\chi^{2}$ & $p$ value \\
\hline \multirow[t]{2}{*}{ Gender } & Male & 184 & 1,078 & 1,262 & 14.58 & \multirow[t]{2}{*}{10.59} & \multirow[t]{2}{*}{0.001} \\
\hline & Female & 8 & 148 & 156 & 5.13 & & \\
\hline \multirow[t]{3}{*}{ Age, years } & $17-35$ & 102 & 588 & 690 & 14.78 & \multirow[t]{3}{*}{1.992} & \multirow[t]{3}{*}{0.369} \\
\hline & $36-45$ & 62 & 423 & 485 & 12.78 & & \\
\hline & $46-65$ & 28 & 215 & 243 & 11.52 & & \\
\hline Site of donation & Campaigns/mobile bus & 94 & 434 & 528 & 17.80 & 13.06 & $<0.001$ \\
\hline \multirow[t]{2}{*}{ Frequency of donation } & First time donor & 111 & 550 & 661 & 16.79 & \multirow[t]{2}{*}{11.169} & \multirow[t]{2}{*}{$<0.001$} \\
\hline & Regular donor & 81 & 676 & 757 & 10.70 & & \\
\hline \multirow[t]{2}{*}{ Type of donation } & Whole blood & 173 & 1,029 & 1,202 & 14.39 & \multirow[t]{2}{*}{4.898} & \multirow[t]{2}{*}{0.027} \\
\hline & Platelet apheresis & 19 & 197 & 216 & 8.80 & & \\
\hline Blood group & $\mathrm{AB}$ & 16 & 70 & 86 & 18.60 & 6.084 & 0.108 \\
\hline
\end{tabular}

\section{Results}

A total of 1,418 samples eligible for blood donation were tested for SARS-CoV-2 IgG antibody. The demographic characteristics are shown in Table 1 . The majority $(88.4 \%)$ of the donors were males and $82.9 \%$ were from age 17 to 45 years. Concerning nationalities, $46.2 \%$ were Indian, followed by $14.8 \%$ from the United Arab Emirates and a small percentage of other 138 nationalities. Concerning frequency of donation, it was found that $53 \%$ of the participants were regular donors, and for site of donation, $62.3 \%$ donated in the Dubai Blood Donation Center while $37.7 \%$ donated at outdoor campaigns (mobile buses or campaigns). The majority were whole blood donors (85.4\%) and $14.6 \%$ were platelet apheresis donors. For the ABO profile, it was found that $41.8 \%$ were $\mathrm{O}$ group, 27\% were B group, 25.2\% were A group, and the $\mathrm{AB}$ group was $6.1 \%$.The demographic factors and the profile of participating donors were found to be similar to our annual donors' data as the participants of this study were selected randomly from eligible donors visiting the center.

All collected samples were tested for COVID IgG antibodies (1,418 samples); 192 (13.5\%) were found to be positive. Then, the demographic profile of 192 donors who were IgG positive was compared with the same of 1,226 donors with negative results, shown in Table 2. It was found that gender, collection site, frequency of dona- tion, and type of donation had significant association with positive test results for SARS-CoV-2 IgG, while age and blood groups had no statistically significant association. The 192 blood donors with positive IgG anti-SARSCoV-2 results were called telephonically and asked further details about few COVID-19 symptoms, exposure factors, and their lifestyle trying to identify related risk factors to COVID-19 infection.

While calling the donors by phone, 165 (85.9\%) donors responded while 27 (14.1\%) donors did not respond. The response of 165 donors to various questions is shown in Table 3 and has been analyzed. It was found that 5.5\% had a history of cough, fever, or flu symptoms during the last 2 months before their current visit to donate blood. $5.5 \%$ have had contact with COVID-19-positive patients, $1.2 \%$ were caring for COVID- 19 patients, and $1.8 \%$ have worked with COVID-19 patients as health care professionals. $6.7 \%$ resided in a containment area. $58.2 \%$ were living in shared accommodations and $38.8 \%$ used shared transportation. $1.2 \%$ attended gathering without PPE and none gave travel history outside the UAE.

When analyzed for risk factor exposure related to lifestyle in donors with positive SARS-CoV-2 IgG as shown in Table 4 , it was found that $68.5 \%$ were exposed to at least 1 risk factor and $38.7 \%$ were exposed to at least 2 risk factors. In addition, it was observed that $33.9 \%$ of the donors were living in shared accommodation and even using shared transportation (2 risk factors at the same time). 
Table 3. Response of 165 donors with positive SARS-CoV-2 IgG to the questionnaire about lifestyle and exposure history

\begin{tabular}{lccc}
\hline Questions asked to donors & Yes & No & Donors having exposure, \% \\
\hline Shared accommodation & 96 & 69 & 58.18 \\
Shared transport & 64 & 101 & 38.79 \\
Reside in a containment area & 11 & 154 & 6.67 \\
Cough, fever & 9 & 156 & 5.45 \\
Contact with COVID-19-positive patient & 9 & 156 & 5.45 \\
Work with COVID-19 samples & 3 & 162 & 1.82 \\
Care for any COVID-19 patient & 2 & 163 & 1.21 \\
Attended gathering without PPE & 2 & 163 & 1.21 \\
Travel outside the UAE & 0 & 165 & 0.00 \\
\hline
\end{tabular}

Table 4. Risk factor exposure for 165 donors with positive SARSCoV-2 IgG

\begin{tabular}{lcr}
\hline Risk factors, $n$ & Donors, $n$ & $\%$ \\
\hline No risk factor & 52 & 31.52 \\
One risk factor & 40 & 24.24 \\
Two risk factors & 64 & 38.79 \\
Three risk factors & 8 & 4.85 \\
Four risk factors & 1 & 0.61 \\
\hline Total & 165 & 100.00 \\
\hline
\end{tabular}

\section{Discussion}

SARS-CoV-2 has spurred a global health crisis. The safety of donors and blood supply has been a concern for blood establishment during the current pandemic.

It is relevant to know the prevalence of SARS-CoV-2 antibodies among asymptomatic blood donors to guide our future decisions concerned with safety of donors, patients, and employees serving in blood establishments. Blood donors' eligibility criteria have been revised and updated periodically during this pandemic aiming to ensure availability and safety of donors and blood supply and to avoid collection from asymptomatic COVID patients.

In this study, the seroprevalence of SARS-CoV-2 IgG antibodies was found in $13.5 \%$ of random donors compared to a reported rate of $0.3-40 \%$ in similar studies from different parts of the world and almost during the same period of time during this pandemic [20-23]. Our prevalence lies below the mean of reported range from different countries worldwide. The United Arab Emirates has implemented precautionary measures to limit the spread of infection early during the pandemic. Social distancing, facial masks, and hand hygiene were required from every individual. All health care services, including blood establishment, were supplied with individual sanitization tunnels, body temperature monitoring, and free PCR test to screen crowded residential areas and health care providers. All previously mentioned measures have played a role in not having a high rate of SARS-CoV-2 IgG antibodies as reported in different parts of the world.

Despite all the additional eligibility criteria, $13.5 \%$ of the eligible donors were seropositive for SARS-CoV-2 IgG. This means that the current eligibility criteria that depend on asking donors few questions about signs and symptoms of COVID-19, travel history, and contacts in addition to routine questions included in the donor history questionnaire are not enough to prevent asymptomatic COVID-19 patients from donating blood and components.

Concerning demographic characteristics, it was found that gender (male), collection site (outdoor campaign), frequency of donation (first time donors), and type of donation (whole blood donation) had significant association with positive SARS-CoV-2 IgG results. The positive results for IgG antibodies were significantly found in males than females ( $p$ value of 0.001 ). Males in our study constitute $88.4 \%$ of participants, which represent the usual percentage of males in our blood donor population. Our finding is similar to that of a large US cohort study showing that males were more likely to test positive for COVID-19 [24], but our findings are different from other similar studies (on blood donors) where results for positive IgG were sex independent [21, 23, $25]$. Other large-scale global studies showed that males and females are at equivalent risk of getting the infection $[24,26]$. 


\section{Concerning Donors and Donations Analysis}

It was found that there is a significant relation between positive IgG test results with donation at outdoor campaigns ( $p$ value of $<0.001$ ), first time donation ( $p$ value of $<0.001)$, and whole blood donation as the type of donation ( $p$ value of 0.027 ). This can be explained by the fact that first time donors are mainly recruited in outdoor campaigns, which are organized in different locations, while regular donors tend to visit the Dubai Blood Donation Center and donate repeatedly. Our regular blood donors are continuously informed and updated about precautions to avoid COVID-19 infection through our smart mobile application and social media. Educational material about COVID-19 signs and symptoms, transmission, and risk factors is given to blood donors before donation and is available in 3 languages (Arabic, English, and Hindi). This may have played a role in having less seroprevalence in regular blood donors. This is similar to what has been reported in a similar study conducted in the USA [23]. Similarly, we found that whole blood donors are significantly showing higher seroprevalence when compared to the regular platelet apheresis donors. This can be explained by the fact that our platelet apheresis donors are donating more frequently (every 2 weeks) and therefore are checked and examined frequently as part of predonation assessment.

The different age groups and blood groups $(\mathrm{ABO})$ had no statistically significance association with positive seroprevalence in this study. Concerning age groups, the participants in our study group were grouped into 3 age groups, with age ranging from 17 to 65 years. No statistically significant association was found between age groups when positive and negative IgG test results were compared, while other studies showed significant correlation of positive IgG antibodies with young population $[21,23,25]$. This can be explained by early implementation of movement restrictions and lock down during this pandemic to all age groups.

No significant relation was found between positive IgG test results and $\mathrm{ABO}$ blood groups which is similar to what has been reported in 1 study in Pakistan [20], while other studies showed that individuals with blood group A have been found to be more at risk as compared to those with blood group $\mathrm{O}[27,28]$. Further studies are required to ensure the significance of such relation.

For nationalities, the Dubai Blood Donation Center receives donors from around 140 nationalities since the expat population is dominated by southeast Asian countries, especially India and Pakistan. In our study, Indians constitute the main group, followed by the United Arab
Emirates nationals, Philippines, and smaller percentages from many other nationalities. All international flights were suspended since the beginning of the pandemic (March-July 2020), and all our donors who participated in this study therefore had no travel history. The significance of positive antibody results and nationalities is of no value in this study as the participants were of varied nationalities, and almost all countries in the world have been affected by this pandemic. In addition, our database does not include ethnic groups of donors which would be more informative than nationalities in terms of genetic predisposition of the immune response to $\mathrm{CO}$ VID-19.

When studying the response of 165 donors who were positive for the IgG antibodies test through a questionnaire, we found that some were having a history of cough, fever, or flu symptoms during the last 2 months preceding their current visit to donate blood, while others have had contact with COVID-19-positive patients or was caring for COVID-19 patients. These findings reiterate the fact that COVID-19 is highly infectious and can lead to asymptomatic infections. All previously mentioned risk factors are listed in our questionnaire and part of eligibility criteria. Noting that our eligibility criteria were limited to a 28-day history of signs, symptoms, or contact history, in this study, we have retrieved the donor history of previously mentioned risk factors to the last 2 months prior to the current donation $[16,17]$. Additional risks included living in shared accommodations, used shared transportation, residing in a containment area, and attending gathering without PPE which are expected lifestyle factors that carry risk of getting COVID-19 infection as it is a contagious disease $[29,30]$.

The role of having one or more risk factors and its effect on the chance of getting infection in donors reflected with a positive IgG antibody test were also analyzed in this study (Table 4). It was noticed that the majority of this group were exposed to at least 1 risk factor and others were exposed to at least 2 risk factors at the same time like, for example, having sharing accommodation and transportation. Several factors contribute to making public transportation stations and vehicle environments high risk for the COVID-19 contagion (UITP 2020): people are confined in limited space. The existence of multiple surfaces, such as seats, handrails, doors, and ticket machines, can easily transfer germs $[30,31]$. This indicates that certain lifestyle criteria could be considered as risk factors to be more prone to infection with COVID-19 infection. 


\section{Limitation of the Study}

In our study, the level of significance for the above risk factors was not calculated as we did not contact all negative IgG donors.

\section{Statement of Ethics}

The study was approved by the Dubai Scientific Research Ethics Committee, DSREC-06/2020_33 dated Jul 21, 2020. Written consent to participate in this study has been taken from all participants.

\section{Conflict of Interest Statement}

All authors declare no conflicts of interest.

\section{Author Contributions}

M.R.: study design, drafting the manuscript, analyzing results and discussion, and final approval. M.R.: drafting material and methods and results related to serological testing and reviewing the manuscript. R.S.: drafting material and methods related to donor eligibility, designing the donors' questionnaire of this study, and reviewing the manuscript. S.K.: data gathering, tables and graph creation, statistical analysis, and reviewing the manuscript. N.T.: gathering information (study questionnaire). R.M.: performing serology testing.

\section{Availability of Data and Materials}

The data that support the findings of this study are not publicly available due to donor information confidentiality but are available from the corresponding author MayY.Raouf(MYRaouf@ dha.gov.ae).

\section{References}

1 World Health Organization. Emergencies preparedness, response. Pneumonia of unknown cause: China. Disease outbreak news. 2020 Jan 5. Available from: https://www.who. $\mathrm{int} / \mathrm{csr} / \mathrm{don} / 05$-january-2020-pneumonia-ofunkown-cause-china/en/.

2 European Centre for Disease Prevention and Control. Cluster of pneumonia cases caused by a novel coronavirus, Wuhan, China. 17 January 2020. Stockholm: ECDC; 2020. Available from: https://www.ecdc.europa.eu/ sites/default/files/documents/Risk\%20assessment $\% 20$ - \% 20 p neum on ia $\% 20$ Wuhan\%20China\%2017\%20Jan\%202020. pdf.

$3 \mathrm{Wu} \mathrm{Z}, \mathrm{McG}$ oogan JM. Characteristics of and important lessons from the coronavirus disease 2019 (COVID-19) outbreak in China: summary of a report of 72314 cases from the Chinese center for disease control and prevention. JAMA. 2020;323:1239-42.

4 World Health Organization. WHO DirectorGeneral's statement on IHR emergency committee on novel coronavirus $(2019-\mathrm{nCoV})$. 2020 Jan 30. Available from: https://www. who.int/director-general/speeches/detail/ who-director-general-s-statement-on-ihremergency-committee-on-novel-coronavirus-(2019-ncov).

5 World Health Organization. WHO DirectorGeneral's opening remarks at the media briefing on COVID-19. 2020 Mar 11. Available from: https://www.who.int/director-general/ speeches/detail/who-director-general-sopening-remarks-at-the-media-briefing-oncovid-19---11-march-2020.

6 World Health Organization. Coronavirus disease (COVID-2019) situation report. Weekly epidemiological update. 2020 Dec 22. Available from: https://www.who.int/emer- gencies/diseases/novel-coronavirus-2019/situation-reports.

7 Zhu N, Zhang D, Wang W, Li X, Yang B, Song J, et al. A novel coronavirus from patients with pneumonia in China, 2019. N Engl J Med. 2020;382(8):727-33.

$8 \mathrm{Xu} \mathrm{Z}$, Shi L, Wang Y, Zhang J, Huang L, Zhang C, et al. Pathological findings of COVID-19 associated with acute respiratory distress syndrome. Lancet Respir Med. 2020; $8(4): 420-2$.

9 Huang C, Wang Y, Li X, Ren L, Zhao J, Hu Y, et al. Clinical features of patients infected with 2019 novel coronavirus in Wuhan, China. Lancet. 2020;395(10223):497-506.

10 Moghadas SM, Fitzpatrick MC, Sah P, Pandey A, Shoukat A, Singer BH, et al. The implications of silent transmission for the control of COVID-19 outbreaks. Proc Natl Acad Sci USA. 2020;117:17513-5.

11 Tong Z-D, Tang A, Li K-F, Li P, Wang H-L, Yi J-P, et al. Potential presymptomatic transmission of SARS-CoV-2, Zhejiang Province, China, 2020. Emerg Infect Dis. 2020;26:10524.

12 Lan L, Xu D, Ye G, Xia C, Wang S, Li Y, et al. Positive RT-PCR test results in patients recovered from COVID-19. JAMA. 2020; 323(15):1502-3.

13 World Health Organization. WHO recommendations on SARS and blood safety. 2003. Available from: https://www.who.int/csr/ sars/guidelines/bloodsafety/en/.

14 World Health Organization. Guidance on maintaining a safe and adequate blood supply during the coronavirus disease 2019 (COVID-19) pandemic and on the collection of COVID-19 convalescent plasma. WHO; 2020 Jul 10. Available from: https://www.who.int/ publications/i/item/maintaining-a-safe-and- adequate-blood-supply-during-the-pandemic-outbreak-of-coronavirus-disease(covid-19).

$15 \mathrm{Yu} \mathrm{X}$, Yang R. COVID-19 transmission through asymptomatic carriers is a challenge to containment. Influenza Other Respir Viruses. 2020;14(4):474-5.

16 AABB's resources for: FDA's updated information for blood establishments regarding the novel coronavirus (COVID-19) outbreak March 2020.

17 AABB's resources for: FDA's updated information for blood establishments regarding the novel coronavirus (COVID-19) outbreak updated May 2020.

18 Tu Y-F, Chien C-S, Yarmishyn AA, Lin Y-Y, Luo Y-H, Lin Y-T, et al. A review of SARS$\mathrm{CoV}-2$ and the ongoing clinical trials. Int $\mathrm{J}$ Mol Sci. 2020;21(7):657.

19 Centers for Disease Control and Prevention (CDC). Epi Info ${ }^{\text {Tw }}$ for windows, open source epidemiologic statistics for public health. Available from: https://www.cdc.gov/epiinfo/pc.html

20 Younas A, Waheed S, Khawaja S, Imam M, Borhany M, Shamsi T. Seroprevalence of SARS-CoV-2 antibodies among healthy blood donors in Karachi, Pakistan. Transfus Apher Sci. 2020;59(6):102923.

21 Slot E, Hogema BM, Reusken CBEM, Reimerink JH, Molier M, Karregat JHM, et al. Low SARS-CoV-2 seroprevalence in blood donors in the early COVID-19 epidemic in the Netherlands. Nat Commun. 2020;11:5744.

22 Filho LA, Szwarcwald CL, Mateos SdOG, de Leon ACMP, de Andrade Medronho R, Gonçalves Veloso V, et al. Seroprevalence of antiSARS-CoV-2 among blood donors in Rio de Janeiro, Brazil. Rev Saude Publica. 2020;54: 69. 
23 Dodd RY, Xu M, Stramer SL. Change in donor characteristics and antibodies to SARS$\mathrm{CoV}-2$ in donated blood in the US, June-August 2020. JAMA. 2020;324(16):1677-9.

24 Vahidy FS, Pan AP, Ahnstedt H, Munshi Y, Choi HA, Tiruneh Y, et al. Sex differences in susceptibility, severity, and outcomes of coronavirus disease 2019: cross-sectional analysis from a diverse US metropolitan area. PLoS One. 2021;16(1):e0245556.

25 Uyoga S, Adetifa IMO, Karanja HK, Nyagwange J, Tuju J, Wanjiku P, et al. Seroprevalence of anti-SARS-CoV-2 IgG antibodies in Kenyan blood donors. Science. 2021; 371(6524):79-82.
26 Peckham H, de Gruijter NM, Raine C, Radziszewska A, Ciurtin C, Wedderburn LR, et al. Male sex identified by global COVID-19 meta-analysis as a risk factor for death and ITU admission. Nat Commun. 2020;11:6317.

$27 \mathrm{Wu} B B, \mathrm{Gu}$ DZ, Yu JN, Yang J, Shen WQ. Association between $\mathrm{ABO}$ blood groups and COVID-19 infection, severity and demise: a systematic review and meta-analysis. Infect Genet Evol. 2020;84:104485.

28 Zhao J, Yang Y, Huang H, Li D, Gu D, Lu X, et al. Relationship between the $\mathrm{ABO}$ blood group and the coronavirus disease 2019 (COVID-19) susceptibility. Clin Infect. Dis. 2020: ciaa1150.
29 Center for Disease Control and prevention (CDC). Living in shared housing. 2020 Oct. Available from: https://www.cdc.gov/ coronavirus/2019-ncov/daily-life-coping/ shared-housing/index.html.

30 Lundkvist A, Hanson S, Olsen B. Pronounced difference in Covid-19 antibody prevalence indicates cluster transmission in Stockholm, Sweden. Infect Ecol Epidemiology. 2020;10:1.

31 Tirachini A, Cats O. COVID-19 and public transportation: current assessment, prospects, and research needs. J Public Trans. 2020;22(1):1-21 